\title{
Body Lateropulsion With Involvement of the Medial Lemniscus Due to a Caudal Pontine Infarction
}

\author{
Hiromasa Tsuda $^{\text {a, b }}$, Aki Genma ${ }^{\text {a }}$, Masaki Ishihara ${ }^{a}$
}

\begin{abstract}
A 63-year-old man with essential hypertension abruptly developed rightward body lateropulsion (BL) and mild dysesthesia in the right upper extremity. No other neurological abnormalities were observed. Cranial magnetic resonance imaging demonstrated a small infarct lesion in the medial zone of the caudal pons on the left side. Under anti-platelet agent, the patient became asymptomatic within 7 days. $\mathrm{BL}$ is defined as irresistible falling to one side without motor weakness. Damage to the ascending graviceptive pathway (GP) may cause $\mathrm{BL}$, and its precise location remains uncertain. The findings of neurological and magnetic resonance imaging in our patient suggest that GP from the vestibular nuclei to the interstitial nucleus of Cajal may cross the midline in the caudal pons, and may run in the vicinity of the medial lemniscus.
\end{abstract}

Keywords: Ascending graviceptive pathway; Interstitial nucleus of Cajal; Magnetic resonance imaging; Pons; Vestibular nuclei

\section{Introduction}

Body lateropulsion (BL) is defined as irresistible falling to one side without motor weakness [1-4]. BL is caused by damage to the ascending graviceptive pathway (GP). However, because there was only two reported cases of BL due to a caudal pontine infarction in English language literatures, precise location of GP remains uncertain in the caudal pons $[1,2]$. We herein describe a case of BL due to a contralateral caudal pontine infarction. This case is very useful for determinating the location of GP in the caudal pons.

Manuscript accepted for publication May 20, 2015

${ }^{\text {a }}$ Department of Neurology, Tokyo Metropolitan Health and Medical Corporation Toshima Hospital, Japan

${ }^{\mathrm{b}}$ Corresponding Author: Hiromasa Tsuda, Department of Neurology, Tokyo Metropolitan Health and Medical Corporation Toshima Hospital, 33-1, Sakaecho, Itabashi-ku, 173-0015, Tokyo, Japan.

Email: hiromasa_tsuda@tokyo-hmt.jp

doi: http://dx.doi.org/10.14740/jmc2197w

\section{Case Report}

A 63-year-old man with essential hypertension abruptly presented with rightward $\mathrm{BL}$ and mild dysesthesia on the right upper extremity in February 2015. There were no other neurological abnormalities. Laboratory examination was within normal ranges. Chest X-ray finding was normal. Electrocardiogram demonstrated no abnormalities. Cranial magnetic resonance (MR) imaging demonstrated a small infarct lesion in the left-sided median zone of the caudal pons (Fig. 1). Cranial MR angiography finding was normal. Intravenous sodium ozagrel $160 \mathrm{mg} /$ day (anti-platelet agent) was initiated. Thereafter, the patient became gradually asymptomatic within 7 days.

\section{Discussion}

In the English language literatures, there have been only 12 reported cases of BL due to a pontine infarction [1-4]. Among these, Yi et al [1] noted eight cases of BL due to pontine tegmentum infarction. Infarct lesion was detected in the rostral pons in five cases, middle pons in one, middle and caudal pons in one, and caudal pons in one. In all of these cases, BL developed toward the opposite side of lesion, except for only one case of a caudal pontine infarction. Therefore, the authors speculated that GP from the vestibular nuclei to the interstitial nucleus of Cajal might run in the pontine tegmentum, and cross the midline in the caudal pons, just above the level of the vestibular nuclei [1]. Furthermore, Okamura et al [2] reported a case of isolated rightward $\mathrm{BL}$ due to left-sided caudal pontine tegmentum infarction, and agreed with the speculation which was proposed by Yi et al [1].

Tsuda et al noted two case of BL due to a rostral pontine tegmentum infarction [3], and one case secondary to a middle pontine tegmentum infarction [4]. In all of these three cases, BL developed toward the opposite side of pontine infarction. In our patient, rightward BL was caused by a left-sided caudal pontine infarction. Based on these four cases, we have provided further evidence for the speculation that GP might cross the midline in the caudal pons, just above the level of the vestibular nuclei which was proposed by Yi et al [1].

Based on cranial MR imaging analysis in 14 cases of isolated deviation of the subjective visual vertical due to brainstem infarction, Zwergal et al [5] reported that there was evidence for an ipsilateral GP running from the vestibular nuclei 


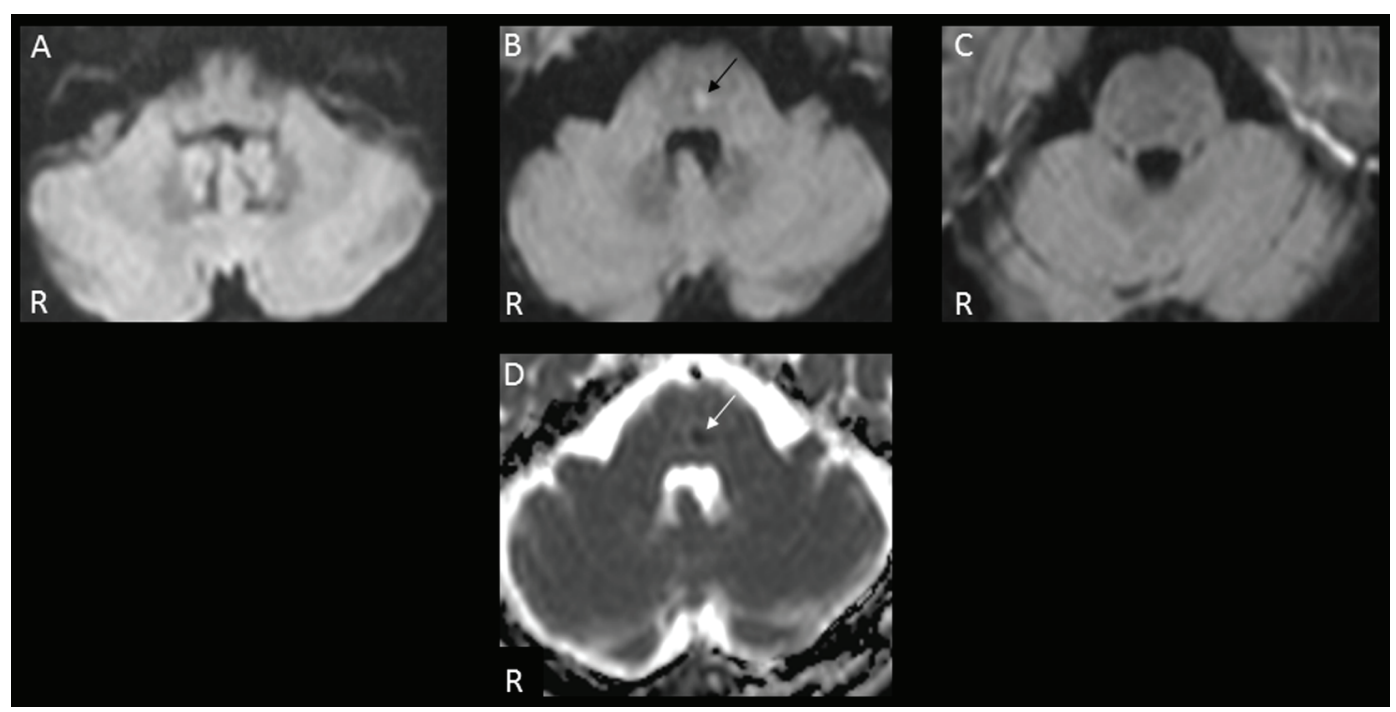

Figure 1. Cranial diffusion-weighted magnetic resonance imaging (A-C: diffusion-weighted image, D: apparent diffusion coefficient) demonstrated a small infarction in the left-sided median zone of the caudal pons (arrow).

close to and within the medial portion of the medial lemniscus. Tsuda and Fujita [4] noted a case of rightward BL and mild dysesthesia on the right upper extremity due to a small infarct lesion in the left middle pontine tegmentum, and speculated that GP might run in the vicinity of the dorsal region to the medial lemniscus in the middle pons. In our patient, based on cranial MR imaging finding, mild dysesthesia on the upper extremity might be induced by partial involvement of the dorsal region of the left-sided medial lemniscus. Consequently, in the middle pons but also the caudal pons, we emphasize that GP may run in the vicinity of the medial lemniscus.

\section{Conflict of Interest}

The authors state that they have no conflict of interest.

\section{Grant Support}

None.

\section{References}

1. Yi HA, Kim HA, Lee H, Baloh RW. Body lateropulsion as an isolated or predominant symptom of a pontine infarction. J Neurol Neurosurg Psychiatry. 2007;78(4):372374.

2. Okamura M, Suzuki K, Komagamine T, Nakamura T, Takekawa H, Asakawa Y, Kawasaki A, et al. Isolated body lateropulsion in a patient with pontine infarction. $\mathrm{J}$ Stroke Cerebrovasc Dis. 2013;22(7):e247-249.

3. Tsuda H, Fujiwara M, Kaneda T. Body lateropulsion with involvement of the medial longitudinal fasciculus due to a rostral pontine tegmentum infarction. J Med Cases. 2014;5(2):55-57.

4. Tsuda H, Fujita T. Body lateropulsion with involvement of the medial leminiscus due to a middle pontine tegmentum infarction. J Med Cases. 2014;5(1):43-44.

5. Zwergal A, Buttner-Ennever J, Brandt T, Strupp M. An ipsilateral vestibulothalamic tract adjacent to the medial lemniscus in humans. Brain. 2008;131(Pt 11):29282935. 